\title{
On Infinite Direct Products of Continuous Unitary One-Parameter Groups
}

\author{
G. Reents \\ Physikalisches Institut, Universität Würzburg, Würzburg, Federal Republic of Germany
}

Received April 4, 1974; in revised form July 29, 1974

\begin{abstract}
We discuss the infinite product of unitary operators in an incomplete direct product of Hilbert spaces. Necessary and sufficient conditions are derived under which this infinite product leads to a continuous unitary one-parameter group provided each factor is assumed to have this property. A certain minimal condition guarantees the existence of a renormalized unitary group. An application is made to product representations of the canonical commutation relations in order to determine the admissible test functions.
\end{abstract}

\section{Introduction}

Our investigations about infinite products of unitary one-parameter groups are motivated by the following physical situation. Consider a system which consists of infinitely many dynamically independent subsystems. The time evolution of the subsystems may be described by unitary operators $U_{r}(t)$ acting in separable Hilbert spaces $H_{r} ; r=1,2, \ldots$ labelling the subsystems. Independence of the various subsystems is achieved most simply if one takes as representation space $H$ for the total system an incomplete direct product of the Hilbert spaces $H_{r}$, $H=\bigotimes_{r}\left(H_{r}, \phi_{r}\right)[1] .\left\{\phi_{r}\right\}_{r=1}^{\infty}$, a sequence of unit vectors with $\phi_{r} \in H_{r}$, which is called reference vector determines a separable subspace of the nonseparable complete direct product. The (naively suggested) time evolution of the total system should then be given by $U(t)=\bigotimes_{r} U_{r}(t)$. However, in general the infinite product of continuous one-parameter groups of unitary operators does not lead to a continuous unitary oneparameter group. $U(t)=\bigotimes_{r} U_{r}(t)$ may happen to be not even unitary in $H=\bigotimes_{r}\left(H_{r}, \phi_{r}\right)$.

So, we are dealing with a family $\left\{U_{r}(\lambda)\right\}_{r=1}^{\infty}$ of unitary operators $U_{r}(\lambda), \lambda \in \mathbb{R}$, acting in separable Hilbert spaces $H_{r}$ such that

$$
U_{r}(\lambda) U_{r}\left(\lambda^{\prime}\right)=U_{r}\left(\lambda+\lambda^{\prime}\right)
$$


and $U_{r}(\lambda)$ is weakly (strongly) continuous in the real parameter $\lambda$. Let us define a truncated product of the $U_{r}(\lambda)$ by

$$
U^{(n)}(\lambda)=\bigotimes_{r} U_{r}^{(n)}(\lambda)
$$

with $U_{r}^{(n)}(\lambda)=U_{r}(\lambda)$ for $r \leqq n$ and $U_{r}^{(n)}(\lambda)=\mathbb{1}_{r}$ for $r>n$. Certainly, $U^{(n)}(\lambda)$ represents a continuous unitary one-parameter group in each incomplete direct product space. This is because $U^{(n)}(\lambda)$ can be written as a finite product of commuting operators each of which having the stated property. In the next Section we shall formulate necessary and sufficient conditions under which $U^{(n)}(\lambda)$ or a subsequence $U^{\left(n_{k}\right)}(\lambda)$ converges strongly to a continuous unitary one-parameter group. We shall see that a certain minimal condition implies the existence of real (renormalization) constants $\left\{\alpha_{r}\right\}_{r=1}^{\infty}$ so that $\bigotimes_{r} U_{r}(\lambda) e^{-i \lambda \alpha_{r}}$ is unitary. In Ref. [2] this formalism has been used to construct a unitary scattering operator for a model with infrared singularities. Section 3 contains an application of our results to determine the admissible test functions for direct product representations of the canonical commutation relations (CCRs). A necessary condition on those test functions given by Woods [3] turns out to be also sufficient.

\section{Relation to Probability Theory and Main Results}

Before going into details we recall some consequences which follow from von Neumann's definition of convergence of an infinite product [1]. Let $\left\{z_{r}\right\}_{r=1}^{\infty}$ be complex numbers, $z_{r}=\left|z_{r}\right| e^{i \theta_{r}}$ with $-\pi<\theta_{r} \leqq \pi$. Then $\Pi z_{r}$ converges to a nonzero value if and only if

(i) $\prod_{r}\left|z_{r}\right|$ converges irrespective of any order of the factors to a nonzero value and

(ii) $\sum_{r}\left|\theta_{r}\right|<\infty$.

Condition (i) is equivalent to $\sum_{r}|1-| z_{r}||<\infty$ and all $z_{r} \neq 0$.

The following lemma states a necessary condition for the convergence of $U^{\left(n_{k}\right)}(\lambda)$. Any subsets $\Delta \subset \mathbb{R}$ occurring are assumed to be Borel sets. By $\mu(\Delta)$ we denote the Lebesgue measure of $\Delta$.

Lemma 2.1. Let $n_{k}, k=1,2, \ldots$, be a subsequence of the positive integers, $n_{k+1}>n_{k}$ for all $k$, and let $U^{\left(n_{k}\right)}(\lambda)$ be given by (1.2). If $U^{\left(n_{k}\right)}(\lambda)$ (with $k \rightarrow \infty$ ) converges strongly in $H=\bigotimes_{r}\left(H_{r}, \phi_{r}\right)$ to a continuous unitary one-parameter group $U(\lambda)$ then there exists a subset $\Delta \subset \mathbb{R}, \mu(\Delta) \neq 0$, such that

$$
\sum_{r}\left(1-\left|\left\langle\phi_{r}, U_{r}(\lambda) \phi_{r}\right\rangle\right|\right)<\infty \quad \text { for all } \lambda \in \Delta
$$


Proof. The group relation (1.1) implies $U_{r}(0)=\mathbb{1}_{r}$ for all $r$ and, therefore, $U(0)=\mathbb{1}$. Because of the continuity of $U(\lambda)$ we have for all $\lambda$ close enough to zero, $\lambda \in \Delta$ say,

$$
\begin{aligned}
\left|\left\langle\bigotimes_{r} \phi_{r}, U(\lambda) \underset{r}{\otimes} \phi_{r}\right\rangle\right| & =\lim _{k \rightarrow \infty} \prod_{r=1}^{n_{k}}\left|\left\langle\phi_{r}, U_{r}(\lambda) \phi_{r}\right\rangle\right| \\
& =\prod_{r=1}^{\infty}\left|\left\langle\phi_{r}, U_{r}(\lambda) \phi_{r}\right\rangle\right| \neq 0 .
\end{aligned}
$$

Note that $\left|\left\langle\phi_{r}, U_{r}(\lambda) \phi_{r}\right\rangle\right| \leqq 1$ for all $r$ and $\lambda \in \mathbb{R}$, thus $\prod_{r}\left|\left\langle\phi_{r}, U_{r}(\lambda) \phi_{r}\right\rangle\right|$ converges irrespective of any ordering. Equation (2.1) is necessary in order that an infinite product converges to a nonzero value [1].

To prove the following theorem strong use is made of the intimate relationship between continuous one-parameter groups of unitary operators and characteristic functions of random variables.

Theorem 2.2. The following conditions are equivalent:

(a) There are real numbers $\left\{\alpha_{r}\right\}_{r=1}^{\infty}$ so that $U_{\text {ren }}^{(n)}(\lambda)=\bigotimes_{r=1}^{n} U_{r}(\lambda) e^{-i \lambda \alpha_{r}} \otimes \mathbb{1}$ converges strongly in $H=\bigotimes\left(H_{r}, \phi_{r}\right)$ to a continuous unitary oneparameter group $U_{\text {ren }}(\lambda)=\bigotimes_{r}^{r} U_{r}(\lambda) e^{-i \lambda \alpha_{r}}$.

(b) There exists a subset $\Delta \subset \mathbb{R}, \mu(\Delta) \neq 0$, and

$$
\sum_{r}\left(1-\left|\left\langle\phi_{r}, U_{r}(\lambda) \phi_{r}\right\rangle\right|\right) \equiv \sum_{r}\left(1-\left|f_{r}(\lambda)\right|\right)<\infty \quad \text { for all } \lambda \in \Delta .
$$

Proof. (a) $\rightarrow$ (b): Is immediate by Lemma 2.1.

(b) $\rightarrow$ (a): Let $f_{r}(\lambda)=\left\langle\phi_{r}, U_{r}(\lambda) \phi_{r}\right\rangle$. Since $f_{r}$ is a continuous function of positive type satisfying $f_{r}(0)=1,\left\{f_{r}\right\}_{r=1}^{\infty}$ may be regarded as characteristic functions of mutually independent random variables $\left\{\sigma_{r}\right\}_{r=1}^{\infty}$ on a probability space ([4], Chapters II and III). The Condition (b) then implies by Theorem 2.7 in Chapter 3 of [5] that the series $\sum_{r}\left(\sigma_{r}-\alpha_{r}\right)$ converges with probability 1 for some sequence of real numbers $\left\{\alpha_{r}\right\}_{r=1}^{\infty}$. Since $f_{r}(\lambda) e^{-i \lambda \alpha_{r}}$ is the characteristic function of $\sigma_{r}-\alpha_{r}$, it follows from Theorem 2.7 in [5] that

$$
\sum_{r}\left|1-f_{r}(\lambda) e^{-i \lambda \alpha_{r}}\right|<\infty \quad \text { for all } \lambda \in \mathbb{R},
$$

the convergence being uniform in every finite interval of $\lambda$. According to Lemma 3.2 of [2] (see also Theorem 3.1 of $[6]) U_{\text {ren }}(\lambda)=\bigotimes_{r} U_{r}(\lambda) e^{-i \lambda \alpha_{r}}$ is unitary in $H=\bigotimes_{r}\left(H_{r}, \phi_{r}\right)$ for all $\lambda \in \mathbb{R}$ and $U_{\text {ren }}(\lambda)=$ strong$\lim _{n \rightarrow \infty} U_{\text {ren }}^{(n)}(\lambda)$. The group relation is obvious by the form of $U_{\text {ren }}(\lambda)$. To 
prove continuity we remark that for any $\Psi \in H$ and any $\varepsilon>0$ there exists $N<\infty$ and $\psi_{N} \in \bigotimes_{r=1}^{N} H_{r}$ such that $\left\|\Psi-\psi_{N} \otimes\left(\bigotimes_{r>N} \phi_{r}\right)\right\|<\varepsilon \quad([7]$, Lemma 3.1). Thus it is sufficient to prove that

$$
\left\langle\bigotimes_{r} \phi_{r}, U_{\text {ren }}(\lambda) \bigotimes_{r} \phi_{r}\right\rangle=\prod_{r} f_{r}(\lambda) e^{-i \lambda \alpha_{r}}
$$

is continuous in $\lambda$, and this follows from the uniform convergence of $\sum_{r}\left|1-f_{r}(\lambda) e^{-i \lambda \alpha_{r}}\right|$.

Remark 2.3. The sequence $\left\{\alpha_{r}\right\}_{r=1}^{\infty}$ is not uniquely determined. Let $\alpha_{r}^{\prime}=\alpha_{r}+\varepsilon_{r}$ and $\sum_{r}\left|\varepsilon_{r}\right|<\infty$. Then $\lim _{n \rightarrow \infty} \bigotimes_{r=1}^{n} U_{r}(\lambda) e^{-i \lambda \alpha_{r}^{\prime}}=e^{-i \lambda \sum \varepsilon_{r}} U_{\text {ren }}(\lambda)$ which is unitary, continuous in $\lambda$ and fulfills (1.1) along with $U_{\text {ren }}(\lambda)$. If, on the other hand, (2.2) holds also true with $\left\{\alpha_{r}\right\}_{r=1}^{\infty}$ replaced by $\left\{\alpha_{r}^{\prime}\right\}_{r=1}^{\infty}$, then we have [1] (equivalence of product vectors) that

$$
\prod_{r}\left\langle U_{r}(\lambda) e^{-i \lambda \alpha_{r}^{\prime}} \phi_{r}, U_{r}(\lambda) e^{-i \lambda \alpha_{r}} \phi_{r}\right\rangle=\prod_{r} e^{i \lambda\left(\alpha_{r}^{\prime}-\alpha_{r}\right)}
$$

exists for all $\lambda \in \mathbb{R}$. By Corollary 2.9 of Ref. [8] we get $\sum_{r}\left|\alpha_{r}^{\prime}-\alpha_{r}\right|<\infty$. Therefore, the generator of $U_{\text {ren }}(\lambda)$ is unique up to a finite additive constant.

The next lemma indicates how to determine the sequence of renormalization constants $\left\{\alpha_{r}\right\}_{r=1}^{\infty}$. Let $f_{r}(\lambda)=\left|f_{r}(\lambda)\right| e^{i \beta_{r}(\lambda)}$. Since $f_{r}(\lambda)$ is continuous in $\lambda$ and $f_{r}(0)=1$ we choose $\beta_{r}(0)=0$ and $\beta_{r}(\lambda)$ continuous as long as $f_{r}(\lambda) \neq 0$.

Lemma 2.4. Let Condition (b) of Theorem 2.2 be fulfilled and let $\beta_{r}(\lambda)$ be as above. Then $\sum_{r}\left|\frac{1}{\lambda} \beta_{r}(\lambda)-\alpha_{r}\right|<\infty$ for every $\lambda \neq 0$ and $\sum_{r}\left|\beta_{r}(\lambda)-\lambda \alpha_{r}\right|$ converges uniformly in $\lambda$ on every bounded interval.

Proof. Condition (b) implies that $\left|f_{r}(\lambda)\right| \longrightarrow r$ uniformly in $\lambda$ on every bounded interval $I$. Let us assume this interval to be centered at zero. Then $f_{r}(\lambda) \neq 0$ and $\beta_{r}(\lambda)$ is continuous in $\lambda$ for $r>N$ and $\lambda \in I$ with some finite $N=N(I)$. Moreover, $\prod_{r>N} f_{r}(\lambda) e^{-i \lambda \alpha_{r}}=\prod_{r>N}\left|f_{r}(\lambda)\right| e^{i\left(\beta_{r}(\lambda)-\lambda \alpha_{r}\right)}$ converges uniformly in $\lambda$ to a nonzero value. This implies uniform convergence of $\prod_{r>N} e^{i\left|\beta_{r}(\lambda)-\lambda \alpha_{r}\right|}=e^{i \sum_{r}\left|\beta_{r}(\lambda)-\lambda \alpha_{r}\right|}$. Now, $\beta_{r}(\lambda)-\lambda \alpha_{r}$ is continuous for $\lambda \in I$ and $r>N$ and $\beta_{r}(\lambda)-\lambda \alpha_{r}=0$ for $\lambda=0$ and all $r$. It follows that $\sum_{r>N}\left|\beta_{r}(\lambda)-\lambda \alpha_{r}\right|$ converges uniformly on $I$. Since $I$ was arbitrary, $\sum_{r}\left|\frac{1}{\lambda} \beta_{r}(\lambda)-\alpha_{r}\right|<\infty$ for every $\lambda \neq 0$. 
According to Remark 2.3 one may especially choose $\alpha_{r}=\beta_{r}(1)$.

The minimal Condition (b) of Theorem 2.2 guarantees the existence of real numbers $\left\{\alpha_{r}\right\}_{r=1}^{\infty}$ such that $\bigotimes_{r} U_{r}(\lambda) \phi_{r}$ is equivalent to $\bigotimes_{r} e^{i \lambda \alpha_{r}} \phi_{r}$. According to Theorem 5.1 of Ref. [3] one would argue that this condition is even sufficient to find a subsequence $n_{k}$ so that $U^{\left(n_{k}\right)}(\lambda)$ converges strongly to a unitary one-parameter group. The last half (sufficiency part) of Theorem 5.1 in [3], however, is incorrect as can be seen from the following.

Theorem 2.5. Let $U^{\left(n_{k}\right)}(\lambda)$ be given by (1.2) and let $\beta_{r}(\lambda)$ be defined as in Lemma 2.4. $U^{\left(n_{k}\right)}(\lambda)$ converges strongly in $H=\bigotimes\left(H_{r}, \phi_{r}\right)$ to a continuous unitary one-parameter group $U(\lambda)$ if and only if

(i) $\sum_{r}\left(1-\left|f_{r}(\lambda)\right|\right)<\infty$ for $\lambda \in \Delta, \mu(\Delta) \neq 0$, and

(ii) $\sum_{k}\left(\sum_{r=n_{k-1}+1}^{n_{k}} \beta_{r}\left(\lambda_{0}\right)\right)$ converges for some $\lambda_{0} \neq 0$.

Proof. Necessity: Lemma 2.1 shows necessity of Condition (i). By Theorem 2.2 and Lemma 2.4 we have that

$$
V^{\left(n_{k}\right)}(\lambda)=\bigotimes_{r=1}^{n_{k}} U_{r}(\lambda) e^{-i \frac{\lambda}{\lambda_{0}} \beta_{r}\left(\lambda_{0}\right)} \otimes \mathbb{1}
$$

converges strongly for all $\lambda$ with any $\lambda_{0} \neq 0$. It follows that

$$
U^{\left(n_{k}\right)}(\lambda) V^{\left(n_{k}\right)}(-\lambda)=e^{i \frac{\lambda}{\lambda_{0}} \sum_{r=1}^{n_{k}} \beta_{r}\left(\lambda_{0}\right)}
$$

converges for all $\lambda$ [note that $U^{\left(n_{k}\right)}(\lambda)$ commutes with all $\left.V^{\left(n_{l}\right)}\left(\lambda^{\prime}\right)\right]$. By Corollary 2.9 of Ref. [8] this implies convergence of $\sum_{k}\left(\sum_{r=n_{k-1}+1}^{n_{k}} \beta_{r}\left(\lambda_{0}\right)\right)$.

Sufficiency: If (i) is satisfied then there exists a sequence $\left\{\alpha_{r}\right\}_{r=1}^{\infty}$ such that $U^{\left(n_{k}\right)}(\lambda) \prod_{r=1}^{n_{k}} e^{-i \lambda \alpha_{r}}$ converges strongly for all $\lambda$ to a continuous unitary one-parameter group. According to Lemma 2.4 and the preceding remark we have $\sum_{r}\left|\beta_{r}(\lambda)-\lambda \alpha_{r}\right|<\infty$ for all $\lambda$, especially for $\lambda=\lambda_{0}$ so so that Condition (ii) implies convergence of $\sum_{k}\left(\sum_{r=n_{k-1}+1}^{n_{k}} \alpha_{r}\right)$. It follows that $\prod_{r=1}^{n_{k}} e^{-i \lambda \alpha_{r}}$ converges to a continuous unitary group $e^{-i \lambda \alpha}$ and we have the desired result.

We turn now to the question whether one can always find a sequence of unit vectors $\left\{\phi_{r}\right\}_{r=1}^{\infty}$ satisfying Condition (b) of Theorem 2.2. 
Theorem 2.6. Given a family of continuous unitary one-parameter groups $\left\{U_{r}(\lambda)\right\}_{r=1}^{\infty}$ acting in Hilbert spaces $H_{r}$ there exists a sequence of unit vectors $\left\{\phi_{r} \in H_{r}\right\}_{r=1}^{\infty}$ such that

$$
\sum_{r}\left(1-\left|\left\langle\phi_{r}, U_{r}(\lambda) \phi_{r}\right\rangle\right|\right) \equiv \sum_{r}\left(1-\left|f_{r}(\lambda)\right|\right)<\infty
$$

for all $\lambda$ of some subset of $\mathbb{R}$ with nonzero Lebesgue measure.

Proof. Let $U_{r}(\lambda)=e^{i \lambda A_{r}}=\int e^{i \lambda x} d E_{x}^{(r)}$ and let $x_{r}$ be a growth point of $E_{x}^{(r)}$, i.e., $E_{x_{r}+\varepsilon}^{(r)}-E_{x_{r}}^{(r)}>0$ for every $\varepsilon>0$. Choose a sequence of positive real numbers $\left\{\varepsilon_{r}\right\}_{r=1}^{\infty}$ such that $\sum_{r} \varepsilon_{r}<\infty$. Let $\phi_{r} \in\left(E_{x_{r}+\varepsilon_{r}}^{(r)}-E_{x_{r}}^{(r)}\right) H_{r}$ and $\left\|\phi_{r}\right\|=1$ for all $r$. Then, for $|\lambda| \leqq 1$,

$$
\begin{aligned}
1-\left|f_{r}(\lambda)\right| & =1-\left|e^{-i \lambda x_{r}} f_{r}(\lambda)\right| \leqq\left|1-e^{-i \lambda x_{r}} f_{r}(\lambda)\right| \\
& \leqq\left|\int\left(1-e^{i \lambda\left(x-x_{r}\right)}\right) d\left\langle\phi_{r}, E_{x}^{(r)} \phi_{r}\right\rangle\right| \\
& \leqq \int_{0}^{\varepsilon_{r}}\left|1-e^{i \lambda y}\right| d\left\langle\phi_{r}, E_{x_{r}+y}^{(r)} \phi_{r}\right\rangle \\
& \leqq \operatorname{Max}_{|\lambda| \leqq 1, y \leqq \varepsilon_{r}} 2\left|\sin \frac{\lambda y}{2}\right| \leqq \varepsilon_{r} .
\end{aligned}
$$

Hence $\sum_{r}\left(1-\left|f_{r}(\lambda)\right|\right)<\infty$ for $|\lambda| \leqq 1$.

It is clear that (2.3) does not determine the sequence $\left\{\phi_{r}\right\}_{r=1}^{\infty}$ uniquely. As can be seen from the construction above it does not even fix the weak equivalence class.

\section{Application to Product Representations of the CCRs}

We want to apply our results to product representations of the CCRs in order to determine the admissible test functions. We consider representations which are infinite direct products of Fock and/or Schrödinger representations of the CCRs. So, let $\left\{V_{r}\right\}_{r=1}^{\infty}$ be a sequence of test function spaces which we choose to be complex Hilbert spaces with finite or enumerably infinite dimension. For $g_{r} \in V_{r}$ let $W_{r}\left(g_{r}\right)$ be the unitary Weyl operators of the Fock or, respectively, Schrödinger representation [9]. Acting in $H_{r}$ the $W_{r}\left(\lambda g_{r}\right)$ are weakly (strongly) continuous in the real parameter $\lambda$ and satisfy the multiplication law

$$
W_{r}\left(g_{r}\right) W_{r}\left(h_{r}\right)=W_{r}\left(g_{r}+h_{r}\right) e^{i\left(g_{r}, h_{r}\right)},
$$

where $\left(g_{r}, h_{r}\right)$ is given by

$$
\left(g_{r}, h_{r}\right)=\frac{1}{2 i}\left\{\left\langle h_{r}, g_{r}\right\rangle-\left\langle g_{r}, h_{r}\right\rangle\right\},
$$


and $\left\langle g_{r}, h_{r}\right\rangle$ denotes the scalar product in $V_{r}$. It follows that for fixed $g_{r}$ and real parameters $\lambda, \lambda^{\prime}$ we have

$$
W_{r}\left(\lambda g_{r}\right) W_{r}\left(\lambda^{\prime} g_{r}\right)=W_{r}\left(\left(\lambda+\lambda^{\prime}\right) g_{r}\right)
$$

for all $r$. Consider the infinite Cartesian product $V^{*}=\underset{r}{\underset{\gamma}{X}} V_{r}$ of the $V_{r}$ consisting of all sequences $g=\left\{g_{r}\right\}_{r=1}^{\infty}$ with $g_{r} \in V_{r}$. Let $V$ be that subspace of $V^{*}$ which consists of all sequences with only finitely many nonvanishing components, $V=\left\{g=\left\{g_{r}\right\}_{r=1}^{\infty}: g_{r}=0\right.$ for almost all $\left.r\right\}$. We extend the bilinear form (3.2) to elements of $V$ by defining

$$
(g, h)=\sum_{r}\left(g_{r}, h_{r}\right)
$$

Let $H=\bigotimes_{r}\left(H_{r}, \phi_{r}\right)$ be an incomplete tensor product of the $H_{r}$ with $\left\|\phi_{r}\right\|=1$ for all $r$. For $g \in V, g=\left\{g_{r}\right\}_{r=1}^{\infty}$ we define in $H$ a representation of the CCRs by

$$
W(g)=\bigotimes_{r} W_{r}\left(g_{r}\right)
$$

Let $\tau$ be the weakest topology on $V$ such that the map $g \rightarrow \bigotimes_{r} W_{r}\left(\lambda g_{r}\right) \phi_{r}$ is norm-continuous for all $\lambda \in \mathbb{R}$. Since the representation $W(g)$ is irreducible, $\tau$ coincides with the weakest vector topology on $V$ such that $g \rightarrow W(g)$ is continuous in the strong operator topology $[8,10]$. Let $g^{(n)}=\left\{g_{r}^{(n)}\right\}_{r=1}^{\infty} \in V$ be a Cauchy sequence in $\tau$ and let $\Psi=\bigotimes_{r} \psi_{r} \in H$. Then

$$
\begin{aligned}
& \left\|\bigotimes_{r} W_{r}\left(\lambda g_{r}^{(n)}\right) \psi_{r}-\bigotimes_{r} W_{r}\left(\lambda g_{r}^{(m)}\right) \psi_{r}\right\|^{2} \\
& \quad=2\left(1-\operatorname{Re} \prod_{r}\left\langle\psi_{r}, W_{r}\left(-\lambda g_{r}^{(n)}\right) W_{r}\left(\lambda g_{r}^{(m)}\right) \psi_{r}\right\rangle\right) \rightarrow 0
\end{aligned}
$$

for $m, n \rightarrow \infty$ and all $\lambda$. This implies

$$
\left|\left\langle\psi_{r}, W_{r}\left(-\lambda g_{r}^{(n)}\right) W_{r}\left(\lambda g_{r}^{(m)}\right) \psi_{r}\right\rangle\right|=\left|\left\langle\psi_{r}, W_{r}\left(\lambda\left(g_{r}^{(m)}-g_{r}^{(n)}\right)\right) \psi_{r}\right\rangle\right| \rightarrow 1
$$

for all $r$ and $\lambda \in \mathbb{R}$. Let us choose $\psi_{s}=\Omega_{s}$, the Fock (Schrödinger) vacuum for some arbitrary $s$, and $\psi_{r}=\phi_{r}$ for $r \neq s$. Then $\bigotimes_{r} \psi_{r} \in H$ and $[12]$

$$
\left|\left\langle\psi_{s}, W_{s}\left(\lambda\left(g_{s}^{(m)}-g_{s}^{(n)}\right)\right) \psi_{s}\right\rangle\right|=\exp \left(-\frac{1}{2} \lambda^{2}\left\|g_{s}^{(m)}-g_{s}^{(n)}\right\|^{2}\right) .
$$

Thus $g_{s}^{(n)}$ converges in the strong topology on $V_{s}$. Since $s$ was arbitrary it follows that there is a canonical map $\sigma$ from $\bar{V}(\tau)$ (the sequence completion of $V$ in the topology $\tau$ ) into $V^{*}$ such that if $\lim g^{(n)}=\hat{g}(\tau)$ with $g^{(n)} \in V$, then $\sigma(\hat{g})=g=\left\{g_{r}\right\}_{r=1}^{\infty} \in V^{*}$. 
Theorem 3.1. Let $V, W(g), \Phi=\bigotimes_{r} \phi_{r}$ and $\bar{V}(\tau)$ be as above. Let $g=\left\{g_{r}\right\}_{r=1}^{\infty} \in V^{*}$. Then $g \in \sigma(\bar{V}(\tau))$ if and only if

$$
\sum_{r}\left(1-\left|\left\langle\phi_{r}, W_{r}\left(\lambda g_{r}\right) \phi_{r}\right\rangle\right|\right) \equiv \sum_{r}\left(1-\left|f_{r}(\lambda)\right|\right)<\infty
$$

for all $\lambda$ of some subset $\Delta \subset \mathbb{R}$ with $\mu(\Delta) \neq 0$.

Proof. Necessity of Condition (3.6) has been shown by Woods [3]. To prove sufficiency we note that according to Theorem 2.2 there is a sequence $\left\{\alpha_{r}\right\}_{r=1}^{\infty}$ of real numbers $\alpha_{r}$ such that $W(\lambda \hat{g})=\bigotimes_{r} W_{r}\left(\lambda g_{r}\right) e^{-i \lambda \alpha_{r}}$ represents a continuous unitary one-parameter group in $H$. Moreover, $W(\lambda \hat{g})=\mathrm{s}-\lim _{n \rightarrow \infty} \bigotimes_{r=1}^{n} W_{r}\left(\lambda g_{r}\right) e^{-i \lambda \alpha_{r}} \otimes \mathbb{1}$ for all $\lambda$. If $\sum_{r}\left|\alpha_{r}\right|<\infty$ then according to Remark 2.3 we may choose $\alpha_{r}=0$ for all $r$. Defining $g^{(n)}$ $=\left\{g_{r}^{(n)}\right\}_{r=1}^{\infty}$ by $g_{r}^{(n)}=g_{r}$ for $r \leqq n, g_{r}^{(n)}=0$ for $r>n$, we have $g^{(n)} \in V$ for all $n$, $g^{(n)} \rightarrow \hat{g}(\tau)$ and $\sigma(\hat{g})=g$. If $\sum_{r}\left|\alpha_{r}\right|$ diverges we denote the partial sums by $\gamma^{(n)}, \gamma^{(n)}=\sum_{r=1}^{n} \alpha_{r}$. Because $\sum_{r}\left|\alpha_{r}\right|$ diverges there is a subsequence $\left\{\alpha_{r_{l}}\right\}_{l=1}^{\infty}$ $\left(r_{l+1}>r_{l}\right.$ for all $\left.l\right)$ of only positive or negative terms such that $\sum_{l} \alpha_{r_{l}}$ diverges. Hence for every $n$ there are finite integers $L(n), K(n)$, $K(n) \geqq L(n)>n$, such that $\left|\sum_{l=L(n)}^{K(n)} \alpha_{r_{l}}\right| \geqq\left|\gamma^{(n)}\right|$. It follows that we can find real numbers $x^{(n)}$ with $\left|x^{(n)}\right| \leqq 1$ and $x^{(n)} \sum_{l=L(n)}^{K(n)} \alpha_{r_{l}}=\sum_{l=L(n)}^{K(n)} x^{(n)} \alpha_{r_{l}}=-\gamma^{(n)}$. Define $g^{(n)}=\left\{g_{r}^{(n)}\right\} \in^{\prime} V$ by $g_{r}^{(n)}=g_{r}$ for $r \leqq n, g_{r_{l}}^{(n)}=x^{(n)} g_{r_{l}}$ for $l=L(n), \ldots$, $K(n)$, and $g_{r}^{(n)}=0$ elsewhere. To compute

$$
\begin{aligned}
\|\left(\bigotimes_{r} W_{r}\left(\lambda g_{r}^{(n)}\right)\right. & -W(\lambda \hat{g})) \Phi \|^{2} \\
& =2\left(1-\operatorname{Re} \prod_{r}\left\langle\phi_{r}, W_{r}\left(\lambda\left(g_{r}-g_{r}^{(n)}\right)\right) e^{-i \lambda \alpha_{r}} \phi_{r}\right\rangle\right)
\end{aligned}
$$

let $I(n)=\left\{r \in \mathbb{N}: r>n, r \neq r_{l}\right.$ for $\left.l=L(n), \ldots, K(n)\right\}$. Then the infinite product becomes

$$
\begin{aligned}
& \prod_{r}\left\langle\phi_{r}, W_{r}\left(\lambda\left(g_{r}-g_{r}^{(n)}\right)\right) e^{-i \lambda \alpha_{r}} \phi_{r}\right\rangle \\
&=\prod_{l=L(n)}^{K(n)}\left|f_{r_{l}}\left(\lambda\left(1-x^{(n)}\right)\right)\right| \prod_{r \in I(n)} f_{r}(\lambda) e^{-i \lambda \alpha_{r}} \\
& \cdot \exp \left(i\left(-\lambda \gamma^{(n)}+\sum_{l=L(n)}^{K(n)}\left\{\beta_{r_{l}}\left(\lambda\left(1-x^{(n)}\right)\right)-\lambda \alpha_{r_{l}}\right\}\right)\right)
\end{aligned}
$$


where $\beta_{r}(\lambda)$ is defined as in Lemma 2.4. Now, by Condition (3.6) $\prod_{r} f_{r}(\lambda) e^{-i \lambda \alpha_{r}}$ converges uniformly in every bounded interval of $\lambda$ (see Theorem 2.2). This implies that

and

$$
\prod_{r \in I(n)} f_{r}(\lambda) e^{-i \lambda \alpha_{r}} \underset{n}{\longrightarrow} 1
$$

$$
\prod_{l=L(n)}^{K(n)}\left|f_{r_{l}}\left(\lambda\left(1-x^{(n)}\right)\right)\right| \underset{n}{\longrightarrow} 1
$$

for all $\lambda$ [note that $K(n) \geqq L(n)>n$ and $\left|x^{(n)}\right| \leqq 1$ for all $\left.n\right]$. Considering the third factor we remark that

$$
\begin{aligned}
-\lambda \gamma^{(n)}+\sum_{l=L(n)}^{K(n)}\left(\beta_{r_{l}}\right. & \left.\left(\lambda\left(1-x^{(n)}\right)\right)-\lambda \alpha_{r_{l}}\right) \\
& =\sum_{l=L(n)}^{K(n)}\left(\beta_{r_{l}}\left(\lambda\left(1-x^{(n)}\right)\right)-\lambda\left(1-x^{(n)}\right) \alpha_{r_{l}}\right)
\end{aligned}
$$

and this tends to zero by Lemma 2.4. Hence

$$
\left\|\left(\bigotimes_{r} W_{r}\left(\lambda g_{r}^{(n)}\right)-W(\lambda \hat{g})\right) \Phi\right\| \underset{n}{\longrightarrow} 0
$$

for all $\lambda$, i.e., $g^{(n)} \rightarrow \hat{g}(\tau)$ and, clearly, $\sigma(\hat{g})=g$.

As a final remark we note that if $\sigma\left(\hat{g}_{1}\right)=\sigma\left(\hat{g}_{2}\right)$ then $W\left(\lambda \hat{g}_{2}\right)=e^{i \lambda \alpha}$ - $W\left(\lambda \hat{g}_{1}\right)$ for some $\alpha \in \mathbb{R}$. This is because $W\left(\lambda \hat{g}_{2}\right) W\left(-\lambda \hat{g}_{1}\right)$ commutes with all $W(f)$ where $f \in V$, for only the weak limit $\sigma\left(\hat{g}_{2}-\hat{g}_{1}\right)=0$ enters in the bilinear form (3.4). Irreducibility and unitarity imply $W\left(\lambda \hat{g}_{2}\right)$ . $W\left(-\lambda \hat{g}_{1}\right)=e^{i \chi(\lambda)}$ or $W\left(\lambda \hat{g}_{2}\right)=e^{i \chi(\lambda)} W\left(\lambda \hat{g}_{1}\right)$. Now, the 1.h.s. and the second factor of the r.h.s. are continuous unitary groups and, therefore, $\chi(\lambda)=\lambda \alpha$ for some $\alpha \in \mathbb{R}$.

Acknowledgement. I would like to thank K. Kraus for useful discussions and for reading the manuscript.

\section{References}

1. von Neumann, J.: Comp. Math. 6, 1 (1938)

2. Reents, G.: J. Math. Phys. 15, 31 (1974)

3. Woods, E. J.: Commun. math. Phys. 17, 1 (1970)

4. Gelfand,I.M., Wilenkin, N.J.: Verallgemeinerte Funktionen IV. Berlin: VEB Deutscher Verlag der Wissenschaften 1964

5. Doob, J. L.: Stochastic Processes. New York: John Wiley 1953

6. Nakagami, Y.: Kōdai Math. Sem. Rep. 22, 341-354 (1970) 
